\title{
ON SECOND ORDER IMPULSIVE FUNCTIONAL DIFFERENTIAL EQUATIONS IN BANACH SPACES
}

\author{
M. BENCHOHRA \\ Université de Sidi Bel Abbés \\ Département de Mathématiques \\ BP 89, 22000 Sidi Bel Abbés, Algérie \\ E-mail: benchohra@yahoo.com \\ S.K. NTOUYAS \\ University of Ioannina \\ Department of Mathematics \\ 45110 Ioannina, Greece \\ E-mail: sntouyas@cc.uoi.gr
}

(Received July, 2000; Revised June, 2001)

In this paper, a fixed point theorem due to Schaefer is used to investigate the existence of solutions for second order impulsive functional differential equations in Banach spaces.

Key words: Impulsive Functional Differential Equations, Fixed Point, Banach Space.

AMS subject classifications: $34 \mathrm{~A} 37,34 \mathrm{G} 20,34 \mathrm{~K} 25$.

\section{Introduction}

This paper is concerned with the existence of solutions for the initial value problem for the second order functional differential equations with impulsive effects as

$$
\begin{gathered}
y^{\prime \prime}=f\left(t, y_{t}\right), t \in J=[0, T], t \neq t_{k}, k=1, \ldots, m . \\
\left.\Delta y\right|_{t=t_{k}}=I_{k}\left(y\left(t_{k}^{-}\right)\right), k=1, \ldots, m, \\
\left.\Delta y^{\prime}\right|_{t=t_{k}}=\bar{I}_{k}\left(y\left(t_{k}^{-}\right)\right), \quad k=1, \ldots, m, \\
y(t)=\phi(t), \quad t \in[-r, 0], y^{\prime}(0)=y_{0}
\end{gathered}
$$

where $f: J \times C([-r, 0], E) \rightarrow E$ is a given function, $(0<r<\infty), 0=t_{0}<t_{1}<\ldots$ $<t_{m}<t_{m+1}=T, \quad I_{k}, \bar{I}_{k} \in C(E, E) \quad(k=1,2, \ldots, m) \quad$ are $\quad$ bounded, $\quad y_{0} \in E$, $\left.\Delta y\right|_{t=t_{k}}=y\left(t_{k}^{+}\right)-y\left(t_{k}^{-}\right),\left.\quad \Delta y^{\prime}\right|_{t=t_{k}}=y^{\prime}\left(t_{k}^{+}\right)-y^{\prime}\left(t_{k}^{-}\right) \quad$ and $y\left(t_{k}^{+}\right), \quad y^{\prime}\left(t_{k}^{-}\right)$and $y^{\prime}\left(t_{k}^{+}\right)$ represent the left and right limits of $y(t)$ and $y^{\prime}(t)$ respectively at $t=t_{k}$, and $E$ is a real Banach space with norm $|\cdot|$.

For any continuous function $y$ defined on the interval $[-r, T]$ and any $t \in J$, we denote by $y_{t}$ the element of $C([-r, 0], E)$ defined by 


$$
y_{t}(\theta)=y(t+\theta), \quad \theta \in[-r, 0] .
$$

Here $y_{t}(\cdot)$ represents the history of the state from time $t-r$, up to the present time $t$.

Impulsive differential equations have become more important in recent years in some mathematical models of real world phenomena, especially in the biological or medical domain (see the monographs of Bainov and Simeonov [2], Lakshmikantham, Bainov, and Simeonov [9], and Samoilenko and Perestyuk [12], and the papers of Agur, Cojocaru, Mazur, Anderson and Danon [1], Goldbeter, Li and Dupont [5]).

Recently an extension to functional differential equations with impulsive effects has been done by Yujun in [16] by using the coincidence degree theory. For other results on functional differential equations, we refer the interested reader to the monograph of Erbe, Qingai and Zhang [4], Hale [6], Henderson [7], and the survey paper of Ntouyas [11].

The fundamental tools used in the existence proofs of all the above mentioned works are essentially fixed point arguments, nonlinear alternative, topological transversality [3], degree theory [10] or the monotone method combined with upper and lower solutions [8].

This paper will be divided into three sections. In Section 2, we will recall briefly some basic definitions and preliminary facts which will be used throughout Section 3. In Section 3

we shall establish an existence theorem for (1.1)-(1.4). Our approach is based on a fixed point theorem due to Schaefer [13] (see also, Smart [14]).

\section{Preliminaries}

In this section, we introduce notations, definitions, and preliminary facts which are used throughout this paper.

$C([-r, 0], E)$ is the Banach space of all continuous functions from $[-r, 0]$ into $E$ with the norm

$$
\|\phi\|=\sup \{|\phi(\theta)|:-r \leq \theta \leq 0\} .
$$

By $C(J, E)$ we denote the Banach space of all continuous functions from $J$ into $E$ with the norm

$$
\|y\|_{J}:=\sup \{|y(t)|: t \in J\} .
$$

A measurable function $y: J \rightarrow E$ is Bochner integrable if and only if $|y|$ is Lebesgue integrable. (For properties of the Bochner integral, see for instance, Yosida [15]).

$L^{1}(J, E)$ denotes the Banach space of functions $y: J \rightarrow E$ which are Bochner integrable normed by

$$
\|y\|_{L^{1}}=\int_{0}^{T}|y(t)| d t \text { for all } y \in L^{1}(J, E) .
$$

In order to define the solution of (1.1)-(1.4), we shall consider the following space

$$
\Omega^{1}=\left\{y:[-r, T] \rightarrow E: y_{k} \in W^{2,1}\left(J_{k}, E\right), k=0, \ldots, m\right.
$$

and there exist

$$
\begin{gathered}
y\left(t_{k}^{-}\right), y\left(t_{k}^{+}\right), y^{\prime}\left(t_{k}^{-}\right), y^{\prime}\left(t_{k}^{+}\right), k=1, \ldots, m \text { with } y\left(t_{k}^{-}\right)=y\left(t_{k}\right), \\
\left.y^{\prime}\left(t_{k}^{-}\right)=y^{\prime}\left(t_{k}\right), \text { and } y(t)=\phi(t), \forall t \in[-r, 0]\right\}
\end{gathered}
$$


which is a Banach space with the norm

$$
\|y\|_{\Omega^{1}}=\max \left\{\left\|y_{k}\right\|_{W^{2,1}\left(J_{k}\right)}, k=0, \ldots, m\right\},
$$

where $y_{k}, y_{k}^{\prime}$ are the restriction of $y$ and $y^{\prime}$ respectively on $J_{k}=\left(t_{k}, t_{k+1}\right], k=0, \ldots, m$, and $W^{2,1}\left(J_{k}\right)$ denotes the Sobolev class of functions $y_{k}: J_{k} \rightarrow E$ such that $y_{k}^{\prime}$ is absolutely continuous and $y_{k}^{\prime \prime} \in L^{1}\left(J_{k}, E\right)$.

We shall also consider the set

$$
\Omega=\left\{y:[-r, T] \rightarrow E: y_{k} \in C\left(J_{k}, E\right), k=0, \ldots, m\right.
$$

and there exist

$$
\left.y\left(t_{k}^{-}\right), y\left(t_{k}^{+}\right), k=1, \ldots, m \text { with } y\left(t_{k}^{-}\right)=y\left(t_{k}\right) \text { and } y(t)=\phi(t), \quad \forall t \in[-r, 0]\right\}
$$

which is a Banach space with the norm

$$
\|y\|_{\Omega}=\max \left\{\left\|y_{k}\right\|_{J}, k=0, \ldots, m\right\} .
$$

Definition 2.1: A map $f: J \times C([-r, 0], E) \rightarrow E$ is said to be an $L^{1}$-Carathéodory if

(i) $\quad t \mapsto f(t, u)$ is measurable for each $u \in C([-r, 0], E)$;

(ii) $\quad u \mapsto f(t, u)$ is continuous for almost all $t \in J$;

(iii) for each $k>0$, there exists $g_{k} \in L^{1}\left(J, \mathbb{R}_{+}\right)$such that

$$
|f(t, u)| \leq g_{k}(t) \text { for all }\|u\| \leq k \text { and for almost all } t \in J .
$$

So let us start by defining what we mean by a solution of the problem (1.1)-(1.4).

Definition 2.2: A function $y \in \Omega \cap \Omega^{1}$ is said to be a solution of (1.1)-(1.4) if $y$ satisfies the equation $y^{\prime \prime}(t)=f\left(t, y_{t}\right) \quad$ a.e. $\quad$ on $J-\left\{t_{1}, \ldots, t_{m}\right\}$ and the conditions $\left.\Delta y\right|_{t=t_{k}}=I_{k}\left(y\left(t_{k}^{-}\right)\right)$and $\left.\Delta y^{\prime}\right|_{t=t_{k}}=\bar{I}_{k}\left(y\left(t_{k}^{-}\right)\right), k=1, \ldots, m$ and $y^{\prime}(0)=y_{0}$.

Our main result is based on the following.

Lemma 2.3: [13] (See also [14], p. 29). Let $S$ be a convex subset of a normed linear space $X$ and assume $0 \in S$. Let $N: S \rightarrow S$ be a completely continuous operator, and let

$$
\Phi(N)=\{y \in S: y=\lambda N(y) \text { for some } 0<\lambda<1\} .
$$

Then either $\Phi(N)$ is unbounded or $N$ has a fixed point.

We need the following auxiliary result.

Lemma 2.4: If $y \in \Omega \cap \Omega^{1}$ then

$$
\begin{gathered}
y(t)=y(0)+t y^{\prime}(0)+\int_{0}^{t}(t-s) y^{\prime \prime}(s) d s \\
+\sum_{0<t_{k}<t}\left\{\left[y\left(t_{k}^{+}\right)-y\left(t_{k}\right)\right]+\left(t-t_{k}\right)\left[y^{\prime}\left(t_{k}^{+}\right)-y^{\prime}\left(t_{k}\right)\right]\right\} \text { for } t \in J .
\end{gathered}
$$

Proof: Recall that $0=t_{0}<t_{1}<\ldots<t_{m}<t_{m+1}=T$. We first show that 


$$
y(t)=y(0)+\int_{0}^{t} y^{\prime}(s) d s+\sum_{0<t_{k}<t}\left\{\left[y\left(t_{k}^{+}\right)-y\left(t_{k}\right)\right]\right\} \text { for } t \in J .
$$

Suppose $t_{k}<t \leq t_{k+1}$. Then

$$
\begin{gathered}
y\left(t_{1}\right)-y(0)=\int_{0}^{t_{1}} y^{\prime}(s) d s \\
y\left(t_{2}\right)-y\left(t_{1}^{+}\right)=\int_{t_{1}}^{t_{2}} y^{\prime}(s) d s \\
\ldots \\
\ldots \\
y\left(t_{k}\right)-y\left(t_{k-1}^{+}\right)=\int_{t_{k-1}}^{t_{k}} y^{\prime}(s) d s \\
y(t)-y\left(t_{k}^{+}\right)=\int_{t_{k}}^{t} y^{\prime}(s) d s .
\end{gathered}
$$

Adding these inequalities together, we get

$$
y(t)-y(0)-\sum_{i=1}^{k}\left[y\left(t_{i}^{+}\right)-y\left(t_{i}\right)\right]=\int_{0}^{t} y^{\prime}(s) d s .
$$

Hence

$$
y(t)=y(0)+\int_{0}^{t} y^{\prime}(s) d s+\sum_{0<t_{k}<t}\left[y\left(t_{k}^{+}\right)-y\left(t_{k}\right)\right] .
$$

Similarly, we have

$$
y^{\prime}(t)=y^{\prime}(0)+\int_{0}^{t} y^{\prime \prime}(s)+\sum_{0<t_{k}<t}\left[y^{\prime}\left(t_{k}^{+}\right)-y^{\prime}\left(t_{k}\right)\right] .
$$

Substituting (2.3) into (2.2), it is easy to get (2.1).

\section{Main Result}

We are now in a position to state and prove our existence result for the problem (1.1)-(1.4). For the study of this problem, we first list the following hypotheses:

(H1) $\quad f: J \times C([-r, 0], E) \rightarrow E$ is an $L^{1}$ Carathéodory map;

(H2) there exist constants $c_{k}, d_{k}$ such that $\left|I_{k}(y)\right| \leq c_{k},\left|\bar{I}_{k}(y)\right| \leq d_{k}$ $k=1, \ldots, m$ for each $y \in E$;

(H3) $\left|f\left(t, y_{t}\right)\right| \leq p(t) \psi\left(\left\|y_{t}\right\|\right)$ for almost all $t \in J$ and all $y \in C([-r, T], E)$, where $p \in L^{1}\left(J, \mathbb{R}_{+}\right)$and $\psi: \mathbb{R}_{+} \rightarrow(0, \infty)$ is continuous and increasing with 


$$
\int_{0}^{T}(T-s) p(s) d s<\int_{c}^{\infty} \frac{d \tau}{\psi(\tau)}
$$

where $c=\|\phi\|+T\left|y_{0}\right|+\sum_{k=1}^{m}\left[c_{k}+\left(T-t_{k}\right) d_{k}\right]$;

(H4)

for each bounded $B \subseteq C([-r, T], E)$ and for each $t \in J$ the set

$$
\left\{\phi(0)+t y_{0}+\int_{0}^{t}(t-s) f\left(s, y_{s}\right) d s+\sum_{0<t_{k}<t} I_{k}\left(y\left(t_{k}\right)\right)+\left(t-t_{k}\right) \bar{I}_{k}\left(y\left(t_{k}\right)\right): y \in B\right\}
$$

is relatively compact in $E$.

Theorem 3.1: Assume that hypotheses (H1)-(H4) hold. Then the IVP (1.1)-(1.4) has at least one solution on $[-r, T]$.

Proof: Transform the problem into a fixed point problem. Consider the operator $N: \Omega \rightarrow \Omega$ defined by

$$
(N y)(t)=\left\{\begin{array}{cc}
\phi(t), & t \in[-r, 0] \\
\phi(0)+t y_{0}+\int_{0}^{t}(t-s) f\left(s, y_{s}\right) d s & \\
+\sum_{0<t_{k}<t}\left[I_{k}\left(y\left(t_{k}\right)\right)+\left(t-t_{k}\right) \bar{I}_{k}\left(y\left(t_{k}\right)\right)\right], & t \in J .
\end{array}\right.
$$

Remark 3.2: Clearly from Lemma 2.4 the fixed points of $N$ are solutions to (1.1)-(1.4).

We shall show that $N$ satisfies the assumptions of Lemma 2.3. The proof will be given in several steps.

Step 1: $N$ maps bounded sets into bounded sets in $\Omega$.

Indeed, it is enough to show that there exists a positive constant $\ell$ such that for each $y \in B_{q}=\left\{y \in \Omega:\|y\|_{\infty} \leq q\right\}, \quad\|y\|_{\infty}:=\sup \{|y(t)|:-r \leq t \leq T\} \quad$ one $\quad$ has $\|N y\|_{\infty} \leq \ell$.

Let $y \in B_{q}$, then for each $t \in J$ we have

$$
(N y)(t)=\phi(0)+t y_{0}+\int_{0}^{t}(t-s) f\left(s, y_{s}\right) d s+\sum_{0<t_{k}<t}\left[I_{k}\left(y\left(t_{k}\right)\right)+\left(t-t_{k}\right) \bar{I}_{k}\left(y\left(t_{k}\right)\right)\right]
$$

By (H1) we have for each $t \in J$,

$$
\begin{gathered}
|(N y)(t)| \leq\|\phi\|+t\left|y_{0}\right|+\int_{0}^{t}(t-s)\left|f\left(s, y_{s}\right)\right| d s \\
+\sum_{0<t_{k}<t}\left|I_{k}\left(y\left(t_{k}\right)\right)\right|+\left|\left(t-t_{k}\right)\right|\left|\bar{I}_{k}\left(y\left(t_{k}\right)\right)\right| \\
\leq\|\phi\|+t\left|y_{0}\right|+\int_{0}^{t}(t-s)\left|g_{q}(s)\right| d s+\sum_{k=1}^{m}\left[\sup \left\{\left|I_{k}(|y|)\right|:\|y\| \infty \leq q\right\}\right. \\
\left.+\left(T-t_{k}\right) \sup \left\{\left|\bar{I}_{k}(|y|)\right|:\|y\| \infty \leq q\right\}\right] .
\end{gathered}
$$

Then for each $h \in N\left(B_{q}\right)$ we have

$$
\|h\|_{\infty} \leq\|\phi\|+T \| y_{0}\left|+\int_{0}^{T}(T-s)\right| g_{q}(s) \mid d s
$$


$+\sum_{k=1}^{m}\left[\operatorname{su}\left\{\left|I_{k}(|y|)\right|:\|y\|_{\infty} \leq q\right\}+\left(T-t_{k}\right) \sup \left\{\left|\bar{I}_{k}(|y|)\right|:\|y\|_{\infty} \leq q\right\}\right]=\ell$.

Step 2: $N$ maps bounded sets into equicontinuous sets of $\Omega$.

Let $\tau_{1}, \tau_{2} \in J, \tau_{1}<t_{2}$ and $B_{q}=\left\{y \in \Omega\right.$ : $\left.\|y\|_{\infty} \leq q\right\}$ be a bounded set of $\Omega$. Let $y \in B_{q}$. Then

$$
\begin{gathered}
\left|(N y)\left(\tau_{2}\right)-(N y)\left(\tau_{1}\right)\right| \leq\left(\tau_{2}-\tau_{1}\right)\left|y_{0}\right|+\int_{\tau_{1}}^{\tau_{2}}\left|g_{q}(s)\right| d s \\
+\int_{0}^{\tau_{2}}\left(\tau_{2}-\tau_{1}\right)\left|g_{q}(s)\right| d s+\int_{\tau_{1}}^{\tau_{2}}\left|\tau_{1}-s\right|\left|g_{q}(s)\right| d s \\
+\sum_{0 \leq t_{k} \leq \tau_{2}-\tau_{1}}\left|I_{k}\left(y\left(t_{k}\right)\right)\right|+\sum_{0<t_{k}<\tau_{2}-\tau_{1}}\left|\tau_{1}-t_{k}\right|\left|\bar{I}_{k}\left(y\left(t_{k}\right)\right)\right| \\
\quad+\sum_{0<t_{k}<\tau_{2}}\left(\tau_{2}-\tau_{1}\right)\left|\bar{I}_{k}\left(y\left(t_{k}\right)\right)\right| .
\end{gathered}
$$

As $\tau_{2} \rightarrow \tau_{1}$ the right-hand side of the above inequality tends to zero.

The equicontinuity for the case $\tau_{1}<\tau_{2} \leq 0$ and $\tau_{1} \leq 0 \leq \tau_{2}$ is obvious.

Step 3: $N: \Omega \rightarrow \Omega$ is continuous.

Let $\left\{y_{n}\right\}$ be a sequence such that $y_{n} \rightarrow y$ in $\Omega$. Then there is an integer $q$ such that $\left\|y_{n}\right\|_{\infty} \leq q$ for all $n \in \mathbb{N}$ and $\|y\|_{\infty} \leq q$, so $y_{n} \in B_{1}$ and $y \in B_{q}$.

We have then by the dominated convergence theorem

$$
\begin{gathered}
\left\|N y_{n}-N y\right\| \sup _{t \in J}\left[\int_{0}^{t}(t-s)\left|f\left(s, y_{n s}\right)-f\left(s, y_{s}\right)\right| d s\right. \\
\quad+\sum_{0<t_{k}<t}\left\{\left|I_{k}\left(y_{n}\left(t_{k}\right)\right)-I_{k}\left(y\left(t_{k}\right)\right)\right|\right. \\
\left.\left.+\left|t-t_{k}\right|\left|\bar{I}_{k}\left(y_{n}\left(t_{k}\right)\right)-\bar{I}_{k}\left(y\left(t_{k}\right)\right)\right|\right]\right] \rightarrow 0 .
\end{gathered}
$$

Thus $N$ is continuous.

As a consequence of Step 1, Step 2, Step 3 and (H4) together with the Ascoli-Arzela theorem we can conclude that $N: \Omega \rightarrow \Omega$ is completely continuous.

Step 4: Now it remains to show that the set

$$
\Phi(N):=\{y \in \Omega: y \in \lambda N(y), \text { for some } 0<\lambda<1\}
$$

is bounded.

Let $y \in \Phi(N)$. Then $y=\lambda N(y)$ for some $0<\lambda<1$. Thus for each $t \in J$

$$
y(t)=\lambda \phi(0)+\lambda t y_{0}+\lambda \int_{0}^{t}(t-s) f\left(s, y_{s}\right) d s+\lambda \sum_{0<t_{k}<t}\left[I_{k}\left(y\left(t_{k}\right)\right)+\left(t-t_{k}\right) \bar{I}_{k}\left(y\left(t_{k}\right)\right)\right] .
$$


This implies by (H2)-(H3) that for each $t \in J$ we have

$$
|y(t)| \leq\|\phi\|+T\left|y_{0}\right|+\int_{0}^{t}(T-s) p(s) \psi\left(\left\|y_{s}\right\|\right) d s+\sum_{k=1}^{m}\left[c_{k}+\left(T-t_{k}\right) d_{k}\right] .
$$

We consider the function $\mu$ defined by

$$
\mu(t)=\sup \{|y(s)|:-r \leq s \leq t\}, 0 \leq t \leq T .
$$

Let $t^{*} \in[-r, t]$ be such that $\mu(t)=\left|y\left(t^{*}\right)\right|$. If $t^{*} \in[0, T]$, by the previous inequality, we have for $t \in[0, T]$

$$
\mu(t) \leq\|\phi\|+T\left|y_{0}\right|+\int_{0}^{t}(T-s) p(s) \psi(\mu(s)) d s+\sum_{k=1}^{m}\left[c_{k}+\left(T-t_{k}\right) d_{k}\right] .
$$

If $t^{*} \in[-r, 0]$ then $\mu(t)=\|\phi\|$ and the previous inequality holds.

Let us take the right-hand side of the above inequality as $v(t)$, then we have

$$
c=v(0)=\|\phi\|+T\left|y_{0}\right|+\sum_{k=1}^{m}\left[c_{k}+\left(T-t_{k}\right) d_{k}\right], \mu(t) \leq v(t), t \in[0, T],
$$

and

$$
v^{\prime}(t)=(T-t) p(t) \psi(\mu(t)), t \in[0, T] .
$$

Using the nondecreasing character of $\psi$ we get

$$
v^{\prime}(t) \leq(T-t) p(t) \psi(v(t)), \quad t \in[0, T]
$$

This implies for each $t \in J$ that

$$
\int_{v(0)}^{v(t)} \frac{d u}{\psi(u)} \leq \int_{0}^{T}(T-s) p(s) d s<\int_{v(0)}^{\infty} \frac{d u}{\psi(u)} .
$$

This inequality implies that there exists a constant $b=b(T, p, \psi)$ such that $v(t) \leq b, t \in J$, and hence $\mu(t) \leq b, t \in J$. Since for every $t \in[0, T],\left\|y_{t}\right\| \leq \mu(t)$, we have

$$
\|y\|_{\infty}:=\sup \{|y(t)|:-r \leq t \leq T\} \leq b
$$

where $b$ depends only on $T$ and on the functions $p$ and $\psi$. This shows that $\Phi(N)$ is bounded.

Set $X:=\Omega$. As a consequence of Lemma 2.3 we deduce that $N$ has a fixed point which is a solution of (1.1)-(1.4).

\section{References}

[1] Agur, Z., Cojocaru, L., Mazur, G., Anderson, R.M. and Danon, Y.L., Pulse mass measles vaccination across age cohorts, Proc. Nat. Acad. Sci. USA 90 (1993), 1169811702.

[2] Bainov, D.D. and Simeonov, P.S., Systems with Impulse Effect, Ellis Horwood Ltd., Chichister 1989. 
[3] Dugundji, J. and Granas, A., Fixed Point Theory, Monografie Mat. PWN, Warsaw 1982.

[4] Erbe, L.H., Kong, Q. and Zhang, B.G., Oscillation Theory for Functional Differential Equations, Pure and Applied Mathematics, Marcel Dekker 1994.

[5] Goldbeter, A., Li, Y.X. and Dupont, G., Pulsatile signalling in intercellular communications: Experimental and theoretical aspects, Math. Applied to Biology and Medicine, Werz. Pub., Winnipeg, Canada (1993), 429-439.

[6] Hale, J.K., Theory of Functional Differential Equations, Springer-Verlag, New York 1977.

[7] Henderson, J., Boundary Value Problems for Functional Differential Equations, World Scientific, Singapore 1995.

[8] Ladde, G.S., Lakshmikantham, V. and Vatsala, A.S., Monotone Iterative Techniques for Nonlinear Differential Equations, Pitman, Boston 1985.

[9] Lakshmikantham, V., Bainov, D.D. and Simeonov, P.S., Theory of Impulsive Differential Equations, World Scientific, Singapore 1989.

[10] Mawhin, J., Topological Degree Methods in Nonlinear Boundary Value Problems, In: Regional Conf. Series in Math, 40, Amer. Math. Soc, Providence, RI 1979.

[11] Ntouyas, S.K., Initial and boundary value problems for functional differential equations via the topological transversality method: A survey, Bull. Greek Math. Soc. 40 (1998), 3-41.

[12] Samoilenko, A.M. and Perestyuk, N.A., Impulsive Differential Equations, World Scientific, Singapore 1995.

[13] Schaefer, H., Uber die methode der a priori schranken, Math. Ann. 129 (1955), 415-416.

[14] Smart, D.R., Fixed Point Theorems, Cambridge Univ. Press, Cambridge, UK 1974.

[15] Yosida, K., Functional Analysis, 6th edition, Springer-Verlag, Berlin 1980.

[16] Yujun, D., Periodic boundary value problems for functional differential equations with impulses, J. Math. Anal. Appl. 210 (1997), 170-181. 


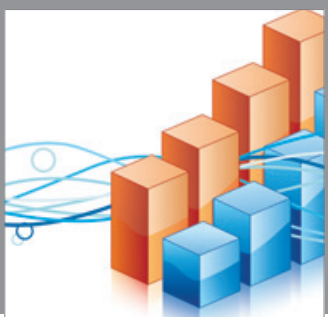

Advances in

Operations Research

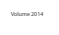

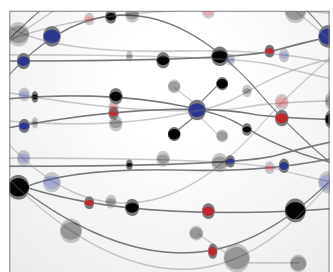

\section{The Scientific} World Journal
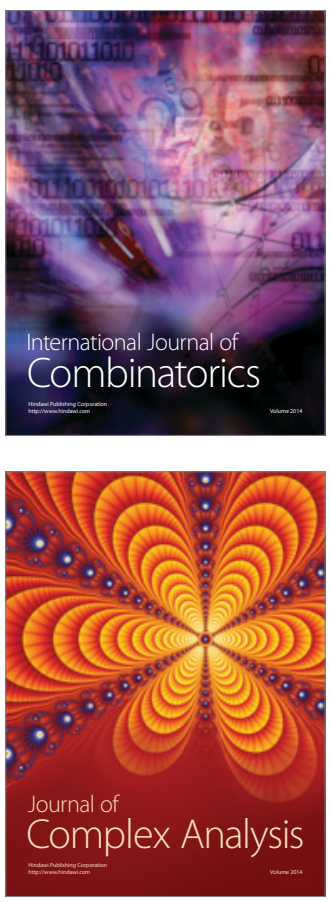

International Journal of

Mathematics and

Mathematical

Sciences
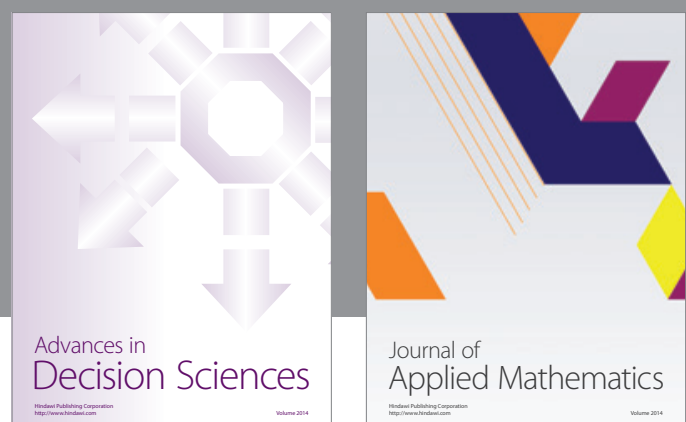

Journal of

Applied Mathematics
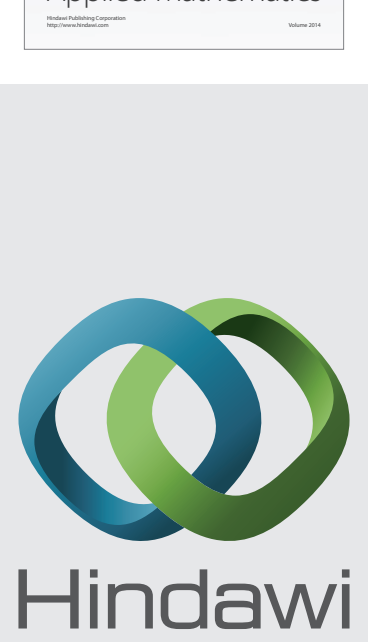

Submit your manuscripts at http://www.hindawi.com
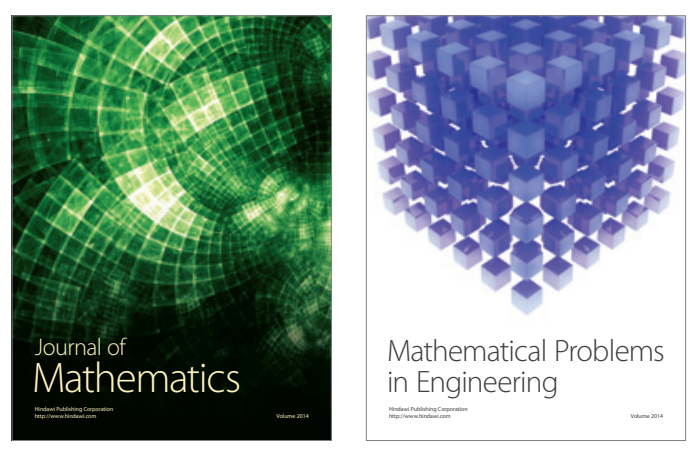

Mathematical Problems in Engineering
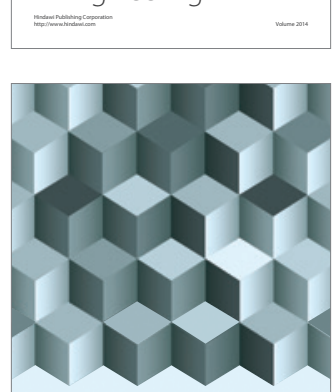

Journal of

Function Spaces
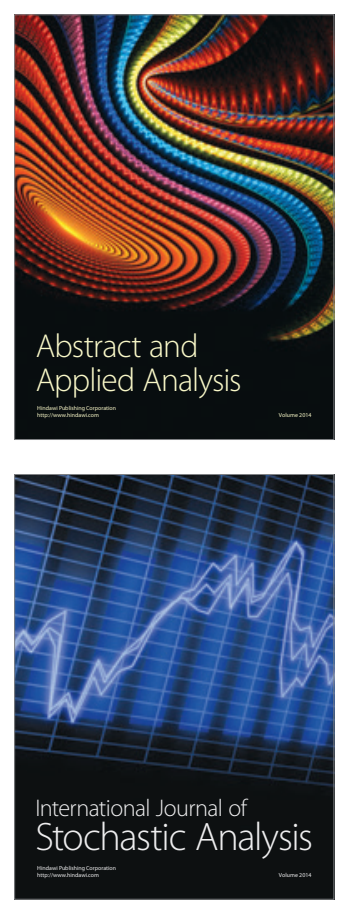

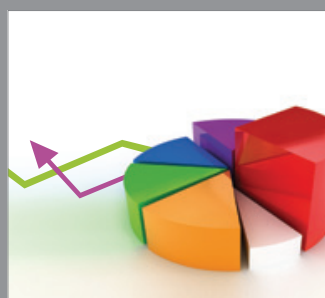

ournal of

Probability and Statistics

Promensencen
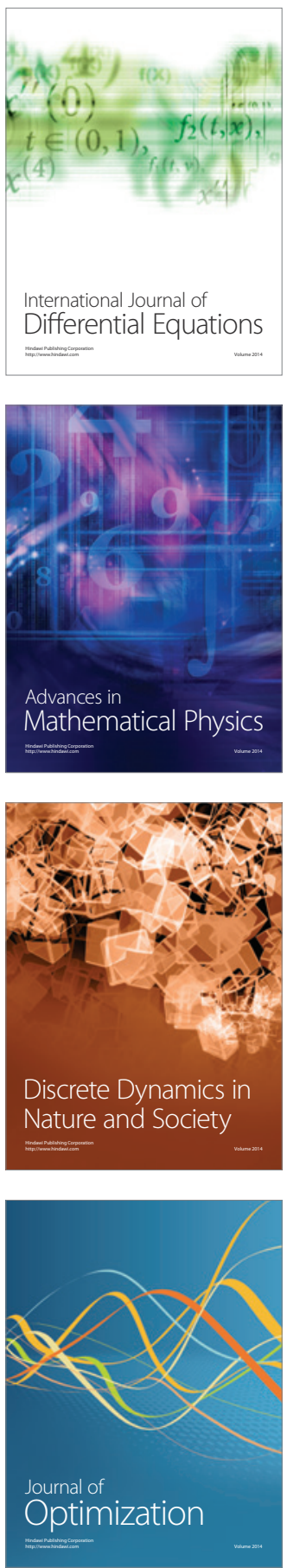